\title{
PARAMÉTRISATIONS DE PETITS CHEMINS EN GÉOMÉTRIE ANALYTIQUE RÉELLE
}

\author{
J.-C. TOUGERON \\ Institut de Mathématiques, Université de Rennes \\ Campus de Beaulieu, 35042 Rennes Cedex, France
}

Soit $X$ un sous-ensemble analytique (ou plus généralement semi-analytique ou sousanalytique) de $\mathbb{R}^{n}$; si $x$ est un point non isolé de $X$, le "lemme du petit chemin" affirme qu'il existe une application analytique $\xi:[0, \varepsilon[\rightarrow X$ telle que $\xi(0)=x$ et $\xi(] 0, \varepsilon[) \subset$ $X \backslash\{x\}$. Ce lemme est très utile dans les démonstrations et on l'étend à des contextes plus généraux, lorsque $X$ est un sous-ensemble analytique d'un certain type d'un ouvert borné de $\mathbb{R}^{n}$ et $x \in \partial X$ ( $X$ peut être une variété pfaffienne, cf. [2], ou $X$ peut être Gevrey au bord, cf. [5]). Dans ce cas, le lemme usuel du petit chemin ne s'applique pas; l'arc que l'on construit est alors une sous-variété connexe et de dimension un de la classe d'ensembles étudiés, mais l'on ne sait rien a priori sur les paramétrisations de cet arc (cependant, dans le cas Gevrey, on sait que l'arc est Gevrey).

Dans cet article $(\S 2)$, on précise la paramétrisation dans un cas très particulier : $X$ est un germe d'ensemble "semi-analytique" à l'origine de $\left(\mathbb{R}^{+}\right)^{n}$ défini à l'aide d'un nombre fini de fonctions de la forme

$$
f\left(x_{1}^{\alpha_{1}}, \ldots, x_{1}^{\alpha_{p}} ; x_{2}^{\alpha_{1}}, \ldots, x_{2}^{\alpha_{p}} ; \ldots ; x_{n}^{\alpha_{1}}, \ldots, x_{n}^{\alpha_{p}}\right)
$$

avec $f$ analytique à valeurs réelles à l'origine de $\mathbb{R}^{n p}$ et $\alpha_{j}$ réels $>0$. Posant $x_{i}=e^{-1 / y_{i}}$, cela revient au même d'étudier au voisinage de 0 les ensembles semi-analytiques définis à l'aide de fonctions analytiques d'exponentielles $e^{-\alpha_{j} / y_{i}}$. La démonstration reprend les idées de [5] : par éclatements, on se ramène au cas où les fonctions sont analytiques en toutes les variables, sauf une; on peut alors appliquer le théorème de préparation. Bien entendu, la recherche d'une paramétrisation nécessite une extension du "lemme de Puiseux"; cette extension est une conséquence facile du théorème de désingularisation $(\S 1)$. Tous les résultats sur les ensembles semi-analytiques usuels s'étendent à cette situation plus générale.

Enfin, dans un dernier paragraphe (§3), on envisage la situation plus générale où l'on

1991 Mathematics Subject Classification: 26B, 26D, 58D.

The paper is in final form and no version of it will be published elsewhere. 
remplace les $x_{i}^{\alpha_{j}}$ par $x_{i}^{\alpha_{j}}\left(\log x_{i}\right)^{\beta_{j}}$; on a des résultats si $n=2$; pour $n>2$, le problème demeure.

\section{Le lemme de Puiseux}

1.1. Soit $\mathcal{O}$ la $\mathbb{R}$-algèbre des germes en 0 de fonctions analytiques $f:] 0, \varepsilon[\rightarrow \mathbb{R}(\varepsilon>0$ dépend de $f$ ); soit $\mathcal{K}$ une sous-algèbre de $\mathcal{O}$ vérifiant les deux conditions suivantes :

(i) $\forall f \in \mathcal{K}^{*}=\mathcal{K} \backslash\{0\}, f$ est non oscillante, i.e. $f$ est strictement monotone dans un intervalle $] 0, \varepsilon[$, donc $f>0$ ou $f<0$.

(ii) $\mathcal{K}$ est un corps.

Si $\mathcal{K}$ est stable par dérivation, la condition (i) équivant à la condition plus faible :

$\left(\mathrm{i}^{\prime}\right) \forall f \in \mathcal{K}^{*}$, on a $f \neq 0$ en tout point d'un intervalle $] 0, \varepsilon[$.

On note $\mathcal{A}$ la sous-algèbre de $\mathcal{K}$ formée des $f$ qui se prolongent par continuité en 0 ; visiblement, $\mathcal{A}$ est un anneau local d'idéal maximal $\mathfrak{m}=\{f \in \mathcal{A}: f(0)=0\} ; \mathcal{K}$ est le corps des fractions de $\mathcal{A}$ et c'est l'union disjointe de $\mathcal{A}$ et $\mathfrak{m}^{-1}=\{f \in \mathcal{K}: 1 / f \in \mathfrak{m}\}$.

On pose $\mathcal{A}_{\mathbb{C}}=\mathcal{A} \otimes_{\mathbb{R}} \mathbb{C} ; \mathcal{K}_{\mathbb{C}}=\mathcal{K} \otimes_{\mathbb{R}} \mathbb{C} ; \mathfrak{m}_{\mathbb{C}}=\mathfrak{m} \otimes_{\mathbb{R}} \mathbb{C} ;$ on définit une relation d'équivalence sur $\mathcal{K}_{\mathbb{C}}^{*}: g \sim f$ si $g / f \in \mathcal{A}_{\mathbb{C}} \backslash \mathfrak{m}_{\mathbb{C}}$, i.e. $g(t) / f(t)$ tend vers une limite finie non nulle quand $t \rightarrow 0$. On note $\nu(f)$ la classe d'équivalence de $f$ (multiplicité de $f$ en $0)$; l'inégalité $\nu(g)>\nu(f)$ signifie que $g / f \in \mathfrak{m}_{\mathbb{C}}$.

\subsection{EXEMPLES}

1.2.1. Supposons que $\mathcal{K}$ est stable par dérivation et vérifie les conditions de 1.1; si $f \in \mathcal{K}$, les corps $\mathcal{K}\left(e^{f}\right)$ ou $\mathcal{K}(F)$ ( $F$ primitive de $f$ ) vérifient les mêmes conditions (d'après Liouville). Il en est de même de la clôture algébrique $\widetilde{\mathcal{K}}$ de $\mathcal{K}$ dans $\mathcal{O}$ (en effet, si $f \in \mathcal{O}^{*}$ vérifie une équation irréductible sur $\mathcal{K}: a_{p} f^{p}+\ldots+a_{0}=0$ avec $a_{i} \in \mathcal{K}$, on a $a_{0} \neq 0$ et $f$ divise $a_{0}$; donc $f \neq 0$ en tout point d'un intervalle $] 0, \varepsilon[$ ). Par ailleurs $f^{\prime}=\left(a_{p}^{\prime} f^{p}+\ldots+a_{0}^{\prime}\right) /\left(p a_{p} f^{p-1}+\ldots+a_{1}\right)$ appartient à $\widetilde{\mathcal{K}}$.

1.2.2. Soit $A$ une sous-algèbre de l'algèbre $\mathcal{E}$ des germes en 0 de fonctions $C^{\infty}$ sur $[0, \varepsilon[$, analytiques sur $] 0, \varepsilon[$, à valeurs réelles; on suppose que $A$ est quasi-analytique, i.e. l'application $A \ni f \rightarrow \widehat{f} \in \mathbb{R}[[t]]$ qui à $f$ associe sa série de Taylor à l'origine, est injective; alors le corps des fractions $\mathcal{K}$ de $A$ vérifie les conditions de 1.1. C'est vrai en particulier si $A$ est l'algèbre $\mathcal{G}$ des germes multisommables dans la direction $\mathbb{R}^{+}$(cf. [5]) : $\mathcal{G}$ est quasi-analytique, stable par dérivation et composition.

1.2.3. Soit $A$ une sous-algèbre quasi-analytique de $\mathcal{E}$, stable par dérivation; alors il existe une sous-algèbre quasi-analytique $\widetilde{A}$ de $\mathcal{E}$, stable par dérivation, telle que $\widetilde{A} \supset A$ et telle que $\widetilde{A} \ni f \rightarrow \widehat{f} \in \mathbb{R}[[t]]$ soit surjective, donc bijective.

Preuve. On considère l'ensemble ordonné par inclusion de toutes les sous-algèbres de $\mathcal{E}$ qui sont quasi-analytiques et stables par dérivation; par l'axiome du choix, il existe un élément maximal $\mathcal{B}$ et il suffit de montrer que $\widehat{\mathcal{B}}=\mathbb{R}[[t]]$; sinon il existerait $\varphi \in \mathbb{R}[[t]] \backslash \widehat{\mathcal{B}}$ et deux cas se présentent :

- Les $\varphi^{(i)}, i \in \mathbb{N}$, sont algébriquement indépendants sur $\widehat{\mathcal{B}}$; si $f \in \mathcal{E}$ avec $\widehat{f}=\varphi$, 
l'algèbre $\mathcal{B}\left[f, f^{\prime}, \ldots\right] \nsupseteq \mathcal{B}$, est quasi-analytique et stable par dérivation, ce qui est contradictoire.

- Les $\varphi^{(i)}, i \in \mathbb{N}$, sont algébriquement dépendants sur $\widehat{\mathcal{B}}$. Soit $p-1$ le plus grand entier tel que $\varphi, \varphi^{\prime}, \ldots, \varphi^{(p-1)}$ soient algébriquement indépendants sur $\widehat{\mathcal{B}}$; soit

$$
\alpha_{q}\left(\varphi, \ldots, \varphi^{(p-1)}\right)\left(\varphi^{(p)}\right)^{q}+\ldots+\alpha_{0}\left(\varphi, \ldots, \varphi^{(p-1)}\right)=0
$$

l'équation de degré minimal $q$ vérifiée $\operatorname{par} \varphi^{(p)}$; les polynômes $\alpha_{i} \in \widehat{\mathcal{B}}\left[y, \ldots, y^{(p-1)}\right]$ se relèvent de manière unique en des polynômes $a_{i} \in \mathcal{B}\left[y, \ldots, y^{(p-1)}\right]$. L'équation différentielle

$$
a_{q}\left(y^{(p)}\right)^{q}+\ldots+a_{0}=0
$$

admet une solution $f \in \mathcal{E}$ telle que $\widehat{f}=\varphi$. En effet, relevons arbitrairement $\varphi$ en $g \in \mathcal{E}$ $(\widehat{g}=\varphi)$ et posons $y=z+g$. On doit trouver une solution infiniment plate à l'origine d'une équation à coefficients dans $\mathcal{E}$,

$$
P\left(z, z^{(1)}, \ldots, z^{(p)}\right)+u_{p} z^{(p)}+u_{p-1} z^{(p-1)}+\ldots+u_{0} z+v_{0}=0
$$

avec $v_{0}$ infiniment plate à l'origine, $P$ polynôme sans termes linéaires ou constants; $\widehat{u}_{p} \neq 0$, car $\widehat{u}_{p}$ est la dérivée par rapport à $\varphi^{(p)}$ du premier membre de $(*)$ et cette dérivée est non nulle, car $q$ est minimal. Sous ces conditions, l'existence d'une solution pour (**) est bien connue.

Soit $\Delta$ le discriminant du polynôme $a_{q}\left(f, \ldots, f^{(p-1)}\right)\left(f^{(p)}\right)^{q}+\ldots+a_{0}\left(f, \ldots, f^{(p-1)}\right)$; on a $\Delta \in \mathcal{B}\left[f, f^{\prime}, \ldots, f^{(p-1)}\right]$ et $\widehat{\Delta} \neq 0$. En dérivant l'équation différentielle vérifiée par $f$, on trouve que $\forall j \geq 1$,

$$
f^{(p+j)}=\frac{P_{j}\left(f, \ldots, f^{(p)}\right)}{\Delta^{n_{j}}}
$$

où $n_{j}$ est un entier $>0$ convenable et $P_{j}$ est un polynôme à coefficients dans $\mathcal{B}$.

Montrons que $\mathcal{B}\left[f, f^{\prime}, \ldots\right]$ est quasi-analytique; soit $g \in \mathcal{B}\left[f, f^{\prime}, \ldots\right]$ telle que $\widehat{g}=0$ et montrons que $g=0$; d'après $(* * *)$, on peut supposer que $g \in \mathcal{B}\left[f, f^{\prime}, \ldots, f^{(p)}\right]$, puis supposer que $g$ est de degré $<q$ en $f^{(p)}$. Comme $\widehat{g}=0$, tous les coefficients de $\widehat{g}$ considéré comme polynôme en $\varphi, \ldots, \varphi^{(p)}$ sont nuls, et donc $g=0$. Ainsi, $\mathcal{B}$ ne serait pas maximale et l'on aboutit là encore à une contradiction.

On en déduit les conséquences suivantes :

- Soient $P_{1}, \ldots, P_{s} \in \widetilde{A}\left[y_{1}, \ldots, y_{r} ; y_{1}^{(1)}, \ldots, y_{r}^{(1)} ; \ldots ; y_{1}^{(p)}, \ldots, y_{r}^{(p)}\right]$. Supposons que le système formel $\widehat{P}_{1}=\ldots=\widehat{P}_{s}=0$ admette une solution formelle $\left(\widehat{y}_{1}(t), \ldots, \widehat{y}_{r}(t)\right) \in$ $\mathbb{R}[[t]]^{r}$; alors cette solution formelle se relève en une solution $\left(y_{1}(t), \ldots, y_{r}(t)\right) \in \widetilde{A}^{r} \mathrm{du}$ système $P_{1}=\ldots=P_{s}=0$. En particulier, les solutions d'un système régulier $y_{j}^{\prime}=$ $P_{j}\left(y_{1}, \ldots, y_{r}\right)\left(1 \leq j \leq r\right.$ et $P_{j}$ polynôme à coefficients dans $\left.\widetilde{A}\right)$ sont dans $\widetilde{A}^{r}$.

- $\widetilde{A}$ est algèbriquement clos dans $\mathcal{E}$; en effet, si $f \in \mathcal{E}$ vérifie une équation $a_{p} f^{p}+\ldots+$ $a_{0}=0$ avec $a_{i} \in \widetilde{A}$, on peut supposer que le discriminant $\Delta$ du polynôme $a_{p} y^{p}+\ldots+a_{0}$ est non nul. Soit $\widetilde{f} \in \widetilde{A}$ tel que $\widehat{\widetilde{f}}=\widehat{f}$; on a $a_{p} \widetilde{f}^{p}+\ldots+a_{0}=0$, donc $f=\widetilde{f}$, car $\widehat{\Delta} \neq 0$. En particulier, si $\varphi \in \mathbb{R}\left\{y_{1}, \ldots, y_{p}\right\}$ est de Nash et si $f_{1}, \ldots, f_{p} \in \widetilde{A}$ avec $f_{i}(0)=0$ pour $i=1, \ldots, p$, on a $\varphi\left(f_{1}, \ldots, f_{p}\right) \in \widetilde{A}$. 
- Soit $\overline{\mathcal{N}}$ la sous-algèbre de $\mathbb{R}\{y\}$ formée des $\varphi$ tels qu'il existe un nombre fini de $\varphi_{1}, \ldots, \varphi_{p} \in \mathbb{R}\{y\}$ avec $\varphi_{1}=\varphi$, les $\varphi_{i}$ étant solutions d'un système

$$
\frac{d \varphi_{i}}{d t}=\frac{P_{i}\left(\varphi_{1}, \ldots, \varphi_{p}\right)}{Q_{i}\left(\varphi_{1}, \ldots, \varphi_{p}\right)}, \quad 1 \leq i \leq p,
$$

avec $P_{i}, Q_{i} \in \mathbb{R}\left[y_{1}, \ldots, y_{p}\right], Q_{i}\left(\varphi_{1}(0), \ldots, \varphi_{p}(0)\right) \neq 0$. Visiblement, $\overline{\mathcal{N}}$ est une algèbre locale qui contient l'algèbre de $\operatorname{Nash} \mathcal{N}$.

Si $f \in \widetilde{A}$ et $f(0)=0$ on a

$$
Q_{i}\left(\varphi_{1} \circ f, \ldots, \varphi_{p} \circ f\right) \frac{d\left(\varphi_{i} \circ f\right)}{d t}=f^{\prime} \cdot P_{i}\left(\varphi_{1} \circ f, \ldots, \varphi_{p} \circ f\right) .
$$

Il en résulte que $\varphi_{i} \circ f \in \widetilde{A}$ pour tout $i$; donc, si $\varphi \in \overline{\mathcal{N}}, \varphi \circ f \in \widetilde{A}$. Enfin, $\widetilde{A}$ est stable par intégration, mais ne l'est pas par composition : si $f \in \widetilde{A}$, en général $f\left(t^{2}\right) \notin \widetilde{A}$.

1.2.4. Soit $\Theta=\left\{\theta_{\alpha}\right\}$ une famille de fonctions, continues et strictement croissantes de $\left[0, \varepsilon\left[\right.\right.$ dans $\mathbb{R}^{+}$, nulles en 0 et analytiques dans $] 0, \varepsilon[$. On suppose que $\Theta$ est stable pour la multiplication; on suppose aussi que $\theta_{\alpha}, \theta_{\beta} \in \Theta$ et $\alpha \neq \beta$ implique que $\theta_{\alpha} / \theta_{\beta}$ ou $\theta_{\beta} / \theta_{\alpha}$ appartient à $\Theta$. On note $\mathcal{H}_{\Theta}$ (resp. $\mathcal{N}_{\Theta}$ ) la sous-algèbre de $\mathcal{O}$ formée des germes en 0 des fonctions qui s'écrivent sous la forme $\varphi\left(\theta_{\alpha_{1}}, \ldots, \theta_{\alpha_{p}}\right)$ avec $\theta_{\alpha_{i}} \in \Theta$ et $\varphi$ analytique (resp. de Nash) au voisinage de l'origine de $\mathbb{R}^{p}$, à valeurs réelles $\left(p, \alpha_{1}, \ldots, \alpha_{p}\right.$ sont variables). D'après le lemme qui suit, $\mathcal{A}=\mathcal{H}_{\Theta}$ ou $\mathcal{N}_{\Theta}$ et son corps des fractions $\mathcal{K}$ vérifient les conditions de 1.1 .

Par exemple, on peut choisir pour $\Theta$ la famille des fonctions $\theta_{\alpha}(t)=t^{\alpha}$ ( $\alpha$ réel $\left.>0\right)$, ou encore la famille des fonctions $\theta_{\alpha}(t)=(1 / t)^{\alpha_{0}}(\log 1 / t)^{\alpha_{1}}\left(\log _{2} 1 / t\right)^{\alpha_{2}} \ldots\left(\log _{q} 1 / t\right)^{\alpha_{q}}$ où $\log _{q}=\log \circ \ldots \circ \log , q$ fois (on choisit les $\alpha_{i} \in \mathbb{R}$ de telle sorte que $\theta_{\alpha}(t) \rightarrow 0$ quand $t \rightarrow 0, t>0)$.

1.3. Lemme. Soient $\theta=\left(\theta_{1}, \ldots, \theta_{p}\right) \in \Theta^{p}, \varphi \in \mathbb{R}\left\{y_{1}, \ldots, y_{p}\right\}$ avec $\varphi(\theta) \neq 0$;il existe des multiindices $\omega_{0} \in \mathbb{N}^{p} ; \omega_{1}, \ldots, \omega_{q} \in \mathbb{Z}^{p} ; a \in \mathbb{R}^{*}$ et $\psi \in \mathbb{R}\left\{y_{1}, \ldots, y_{p} ; z_{1}, \ldots, z_{q}\right\}$, $\psi(0)=0$, tels que $\forall i=1, \ldots, q, \theta^{\omega_{i}} \in \Theta$ et

$$
\varphi\left(\theta_{1}, \ldots, \theta_{p}\right)=a \theta^{\omega_{0}}\left(1+\psi\left(\theta_{1}, \ldots, \theta_{p} ; \theta^{\omega_{1}}, \ldots, \theta^{\omega_{q}}\right)\right)
$$

(On a un résultat analogue si on remplace fonctions analytiques par fonctions de Nash ou si on remplace $\mathbb{R}$ par $\mathbb{C})$.

Preuve. Soit $\nu(\gamma)$ la multiplicité en 0 d'un élément $\gamma \in \Theta$; notons $\mathcal{I}_{\gamma}$ l'idéal de $\mathbb{R}\left[y_{1}, \ldots, y_{p}\right]$ engendré par tous les monômes $y^{\mu}\left(\mu \in \mathbb{N}^{p}\right)$ tels que $\nu\left(\theta^{\mu}\right) \geq \nu(\gamma) ; \mathcal{I}_{\gamma}$ admet un système minimal de générateurs formé de monômes $y^{\mu}, \mu \in \widetilde{\Omega}_{\gamma}\left(\widetilde{\Omega}_{\gamma}\right.$ sous-ensemble fini de $\left.\mathbb{N}^{p}\right)$; ce système est unique et tout monôme appartenant à $\mathcal{I}_{\gamma}$ est multiple d'un tel $y^{\mu}$; on en déduit que l'ensemble $\Omega_{\gamma}$ des $\omega \in \mathbb{N}^{n}$ tels que $\nu\left(\theta^{\omega}\right)=\nu(\gamma)$, i.e. $\theta^{\omega}=\gamma$, est fini et contenu dans $\widetilde{\Omega}_{\gamma}$.

Posons $\varphi=\sum a_{\omega} y^{\omega}$ et notons $\Gamma$ l'ensemble des $\gamma \in \Theta$ tels que $\Omega_{\gamma}$ soit non vide et $\sum_{\omega \in \Omega_{\gamma}} a_{\omega} \neq 0\left(\right.$ donc $\left.\sum_{\omega \in \Omega_{\gamma}} a_{\omega} \theta^{\omega}=\left(\sum_{\omega \in \Omega_{\gamma}} a_{\omega}\right) \cdot \gamma\right)$. Toute suite d'éléments de $\Gamma$ telle que la suite des multiplicités soit strictement décroissante est stationnaire, car la suite des idéaux $\mathcal{I}_{\gamma}$ est alors strictement croissante; $\Gamma$ admet donc un élément $\gamma_{0}=\theta^{\omega_{0}}$ de plus petite multiplicité. 
Soient $\omega_{1}^{\prime}, \ldots, \omega_{q}^{\prime}$ les éléments de $\widetilde{\Omega}_{\gamma_{0}} \backslash \Omega_{\gamma_{0}} ;$ décomposons la série $\sum a_{\omega} y^{\omega}$ sous la forme

$$
\sum_{\omega \in \Omega_{\gamma_{0}}} a_{\omega} y^{\omega}\left(1+\varphi_{\omega}(y)\right)+\sum_{i=1}^{q} a_{\omega_{i}^{\prime}} y^{\omega_{i}^{\prime}}\left(1+\varphi_{\omega_{i}^{\prime}}(y)\right)+\sum_{\omega \in \Omega^{\prime}} a_{\omega} y^{\omega}
$$

$\Omega^{\prime}$ désignant l'ensemble des $\omega \in \mathbb{N}^{n}$ tels que $y^{\omega} \notin \mathcal{I}_{\gamma_{0}} ; \varphi_{\omega}, \varphi_{\omega_{i}^{\prime}}$ appartiennent à $\mathbb{R}\left\{y_{1}, \ldots\right.$ $\left.\ldots, y_{p}\right\}$ et s'annulent en 0 . Si $a=\sum_{\omega \in \Omega_{\gamma_{0}}} a_{\omega}$, on a

$$
\begin{aligned}
\sum a_{\omega} \theta^{\omega} & =a \theta^{\omega_{0}}\left[1+\sum_{\omega \in \Omega_{\gamma_{0}}} a^{-1} \varphi_{\omega}(\theta)+\sum_{i=1}^{q} a_{\omega_{i}^{\prime}} a^{-1} \theta^{\omega_{i}^{\prime}-\omega_{0}}\left(1+\varphi_{\omega_{i}^{\prime}}(\theta)\right)\right] \\
& =a \theta^{\omega_{0}}\left(1+\psi\left(\theta_{1}, \ldots, \theta_{p} ; \theta^{\omega_{1}}, \ldots, \theta^{\omega_{q}}\right)\right)
\end{aligned}
$$

avec $\omega_{i}=\omega_{i}^{\prime}-\omega_{0} \in \mathbb{Z}^{p}$ et

$$
\psi=\sum_{\omega \in \Omega_{\gamma_{0}}} a^{-1} \varphi_{\omega}(y)+\sum_{i=1}^{q} a_{\omega_{i}^{\prime}} a^{-1} z_{i}\left(1+\varphi_{\omega_{i}^{\prime}}(y)\right)
$$

Tout $f \in \mathcal{H}_{\Theta}^{*}$ tel que $f(0)=0$ est donc équivalent à un germe $a \theta\left(a \in \mathbb{R}^{*}, \theta \in \Theta\right)$ et $f$ admet un développement asymptotique convergeant suivant l'échelle $\Theta$, i.e. $f=\sum a_{\alpha} \theta_{\alpha}$ où $a_{\alpha} \in \mathbb{R} ; \theta_{\alpha} \in \Theta$ et $\alpha<\beta$ implique $\nu\left(\theta_{\alpha}\right)<\nu\left(\theta_{\beta}\right)$ (dans l'ordre total sur les $\alpha$, chaque $\alpha$ admet un successeur mais pas toujours un antécédent). Le résultat suivant est une extension du lemme de Puiseux usuel (cf. [3] pour un résultat comparable en formel) :

1.4. ThÉORÈme. Soit $\theta=\left(\theta_{1}, \ldots, \theta_{p}\right) \in \Theta^{p}$ et soit

$$
Q(t, W)=W^{q}+\sum_{i=1}^{q} f_{i}(t) W^{q-i}
$$

un polynôme unitaire à coefficients $f_{i} \in \mathbb{C}\left\{\theta_{1}, \ldots, \theta_{p}\right\}$. Alors il existe un nombre fini de multi-indices $\omega_{1}, \ldots, \omega_{s} \in \mathbb{Q}^{p}$ tels que $\forall j=1, \ldots, s, \theta^{\omega_{j}} \in \Theta^{1 / m}$, pour un certain entier $m \geq 1$; et une fonction analytique $\phi \in \mathbb{C}\left\{y_{1}, \ldots, y_{s}\right\}$, tels que $\phi\left(\theta^{\omega_{1}}, \ldots, \theta^{\omega_{s}}\right)$ soit racine de l'équation $Q(t, W)=0$; en outre, si les $f_{i}$ sont de Nash, $\phi$ est de Nash.

1.5. Corollaire. Soit $\mathcal{K}$ le corps des fractions de $\mathcal{K}_{\Theta}$ ou $\mathcal{N}_{\Theta} ; \mathcal{K}_{\mathbb{C}}$ est algébriquement clos si et seulement si $\theta^{1 / m} \in \Theta, \forall \theta \in \Theta$ et $\forall m$ entier $\geq 1$.

On a aussi le résultat suivant (la preuve est analogue à celle de 1.4) :

1.6. THÉORÈmE. Avec les notations de $1.1, \mathcal{K}_{\mathbb{C}}$ est algébriquement clos si et seulement si les deux conditions suivantes sont satisfaites:

(i) $f_{1}, \ldots, f_{s} \in \mathfrak{m}$ et $\phi \in \mathbb{R}\left\{y_{1}, \ldots, y_{s}\right\}$ de Nash $\Rightarrow \phi\left(f_{1}, \ldots, f_{s}\right) \in \mathcal{A}$.

(ii) $\forall f \in \mathcal{A}, f>0$, et $\forall m$ entier $\geq 1$, on a $f^{1 / m} \in \mathcal{A}$.

1.7. Preuve de 1.4. Soit $w_{0}$ une racine du polynôme complexe $Q(0, W)$; en remplaçant $W$ par $W+w_{0}$, on peut supposer que $f_{q}(0)=0$ et l'on cherche une racine de $Q(t, W)=0$ qui s'annule en $t=0$. Soit $\underline{f}$ l'application $\left[0, \varepsilon\left[\ni t \rightarrow\left(f_{1}(t), \ldots, f_{q}(t)\right) \in \mathbb{C}^{q}\right.\right.$ et posons $a=\underline{f}(0)$; soit $X$ le plus petit germe en $a$ d'ensemble de Nash qui contient la courbe $\underline{f} ; X$ est irréductible. On note $f$ l'application $\left(\mathbb{R}^{+}, 0\right) \ni t \rightarrow \underline{f}(t) \in(X, a)$. 
Le polynôme générique $W^{q}+\sum_{i=1}^{q}\left(z_{i} \mid X\right) W^{q-1}$, à coefficients dans l'algèbre $\mathcal{N}_{X, a}$ des germes de Nash en $a$ sur $X$, a peut être des facteurs multiples. Ce polynôme admet dans $\mathcal{N}_{X, a}[W]$ un facteur $Q^{\prime}$ qui est un polynôme distingué et qui est sans facteurs multiples :

$$
Q^{\prime}(z, W)=W^{q^{\prime}}+\sum_{i=1}^{q^{\prime}} g_{i}(z) W^{q-i}
$$

$\left(q^{\prime}>0\right.$ et $\left.g_{i}(a)=0, \forall i\right)$. On cherche une racine de $Q^{\prime}(f(t), W)=0$.

Soit $\delta \in \mathcal{N}_{X, a}^{*}$ le discriminant de $Q^{\prime}(z, W)$ et soit $X^{\prime}$ le germe de Nash réunion de $\delta=0$ et des points singuliers de $X$. D'après le théorème de désingularisation d'Hironaka, il existe un morphisme de Nash $\pi:\left(\widetilde{X}, \pi^{-1}(a)\right) \rightarrow(X, a)$ vérifiant les conditions suivantes :

(i) $\pi$ est propre et $\widetilde{X}$ est régulier, $\operatorname{dim} \tilde{X}=\operatorname{dim} X=r$.

(ii) $\pi$ induit un difféomorphisme de $\tilde{X} \backslash \pi^{-1}\left(X^{\prime}\right)$ sur $X \backslash X^{\prime}$.

(iii) $\forall \widetilde{a} \in \pi^{-1}(a)$, le germe en $\widetilde{a}$ de $\delta \circ \pi=0$ est réunion de certains hyperplans de coordonnées, pour un système de coordonnées convenables de $\widetilde{X}$ en $\widetilde{a}$.

Par construction de $X$, le germe $f(] 0, \varepsilon[)$ est contenu dans $X \backslash X^{\prime}$ et $\left.f \mid\right] 0, \varepsilon[$ se relève de manière unique en une application $\widetilde{f}:] 0, \varepsilon\left[\rightarrow \widetilde{X} \backslash \pi^{-1}\left(X^{\prime}\right)\right.$; soit $\widetilde{a} \in \pi^{-1}(a)$ une valeur d'adhérence de $\widetilde{f}(t)$ quand $t \rightarrow 0, t>0$.

On peut supposer $a=0$; après plongement de $(\widetilde{X}, \widetilde{a})$ dans $\left(\mathbb{C}^{q}, 0\right)$, l'application $\pi$ : $(\widetilde{X}, \widetilde{a}) \rightarrow(X, 0)$ se relève en une application $\underline{\pi}:\left(\mathbb{C}^{q}, 0\right) \rightarrow\left(\mathbb{C}^{q}, 0\right)$ et l'on sait, par construction de $\pi$, que $\underline{\pi}$ se décompose sous la forme $\underline{\pi}=\pi_{1} \circ \ldots \circ \pi_{\ell}$ où $\pi_{i}:\left(\mathbb{C}^{q}, 0\right) \rightarrow$ $\left(\mathbb{C}^{q}, 0\right)$ a l'une des deux formes suivantes :

a) $\pi_{i}$ est un difféomorphisme de Nash.

b) $\pi_{i}^{*}\left(z_{j}\right)=z_{j}$, pour $1 \leq j \leq k(1 \leq k \leq q) ; \pi_{i}^{*}\left(z_{j}\right)=z_{1}\left(z_{j}+a_{j}\right), a_{j} \in \mathbb{C}$, pour $k+1 \leq j \leq q$.

Démontrons que l'application $\underline{f}:\left[0, \varepsilon\left[\rightarrow \mathbb{C}\right.\right.$ se relève en $F_{i}:\left[0, \varepsilon\left[\rightarrow \mathbb{C}^{q}\right.\right.$ telle que $F_{i}(0)=0$ et $\pi_{1} \circ \ldots \circ \pi_{i} \circ F_{i}=\bar{f}$; montrons en outre l'existence d'un nombre fini de multi-indices $\omega_{1}, \ldots, \omega_{s} \in \mathbb{Z}^{p}$ tels que $\theta^{\omega_{j}} \in \Theta, j=1, \ldots, s$, et celle d'une application analytique $\phi:\left(\mathbb{C}^{s}, 0\right) \rightarrow\left(\mathbb{C}^{q}, 0\right)$, avec $F_{i-1}=\phi\left(\theta^{\omega_{1}}, \ldots, \theta^{\omega_{s}}\right)$.

On procède par induction sur $i$; c'est vrai si $i=1$, car $F_{0}=f$; supposons-le vrai pour $F_{i-1}$ et démontrons-le pour $F_{i}$; on a $\pi_{i} \circ F_{i}=F_{i-1}$; si $\pi_{i}$ a la forme a), le résultat est évident; dans le cas b), on a si $\phi_{1}, \ldots, \phi_{q}$ sont les composantes de $\phi$ et $F_{i, 1}, \ldots, F_{i, q}$ celles de $F_{i}$,

$$
\begin{aligned}
& \text { - } F_{i, j}=F_{i-1, j}=\phi_{j}\left(\theta^{\omega_{1}}, \ldots, \theta^{\omega_{s}}\right), 1 \leq j \leq k, \\
& \text { - } F_{i, j}=\frac{F_{i-1, j}}{F_{i-1,1}}-a_{j}=\frac{\phi_{j}\left(\theta^{\omega_{1}}, \ldots, \theta^{\omega_{s}}\right)}{\phi_{1}\left(\theta^{\omega_{1}}, \ldots, \theta^{\omega_{s}}\right)}-a_{j}, k+1 \leq j \leq q .
\end{aligned}
$$

Si le numérateur de la dernière fraction est non nul, il existe d'après le lemme 1.3 des multi-indices $\omega_{0}, \omega_{s+1}, \ldots, \omega_{s^{\prime}} \in \mathbb{Z}^{p}$ tels que $\theta^{\omega_{s+1}}, \ldots, \theta^{\omega_{s^{\prime}}} \in \Theta ; a \in \mathbb{R}^{*}$ et $\psi \in$ $\mathbb{R}\left\{y_{1}, \ldots, y_{s^{\prime}}\right\}, \psi(0)=0$, avec

$$
F_{i, j}+a_{j}=a \theta^{\omega_{0}}\left(1+\psi\left(\theta^{\omega_{1}}, \ldots, \theta^{\omega_{s^{\prime}}}\right)\right)
$$

Mais d'après le choix de $\widetilde{a}$, il existe des $t_{\nu}>0, t_{\nu} \rightarrow 0$ tels que $F_{i, j}\left(t_{\nu}\right) \rightarrow 0$; donc si $a_{j} \neq 0, \theta^{\omega_{0}}=1$ et $a_{j}=a$; si $a_{j}=0, \theta^{\omega_{0}} \in \Theta$. Si le numérateur est nul, on a $a_{j}=0$ 
et $F_{i, j}=0$. Ainsi $F_{i}$ a les mêmes propriétés que $F_{i-1}$ et la preuve par induction est terminée.

L'application $\underline{f}$ se relève donc en une application $\underline{\tilde{f}}=F_{l}$ telle que $\underline{\tilde{f}}(0)=0$ et $\pi \circ \underline{\tilde{f}}=\underline{f}$; en outre $\underline{\tilde{f}}$ est de la forme $\phi\left(\theta^{\omega_{1}}, \ldots, \theta^{\omega_{s}}\right), \omega_{j} \in \mathbb{Z}^{p}$ et $\theta^{\omega_{j}} \in \Theta, \phi$ analytique. Supposons que le plongement de $(\widetilde{X}, \widetilde{a})$ dans $\left(\mathbb{C}^{q}, 0\right)$ identifie $(\widetilde{X}, \widetilde{a})$ à $\left(\mathbb{C}^{r} \times\{0\}, 0\right)$; posons

$$
\widetilde{f}=\left(\underline{\tilde{f}}_{1}, \ldots, \underline{\tilde{f}}_{r}\right) ; \quad \widetilde{g}_{i}=g_{i} \circ \pi ; \quad \widetilde{Q}^{\prime}(z, W)=W^{q^{\prime}}+\sum_{i=1}^{q^{\prime}} \widetilde{g}_{i}(z) W^{q^{\prime}-i}
$$

On est ramené à chercher une racine de $\widetilde{Q}^{\prime}(\widetilde{f}(t), W)=0$.

Si $\widetilde{\delta}$ est le discriminant de $\widetilde{Q}^{\prime}(z, W)$, l'ensemble $\widetilde{\delta}=0$ est contenu dans l'ensemble $z_{1} \ldots z_{r}=0$; il est bien connu que les racines de $\widetilde{Q}^{\prime}$ sont de la forme $\psi\left(z_{1}^{1 / m}, \ldots, z_{r}^{1 / m}\right)$ avec $\psi$ de Nash, $m$ est un entier $>0$ qu'on peut choisir égal à $q^{\prime}$ ! Si

$$
\underline{\tilde{f}}_{j}=a_{j} \theta^{\omega_{0, j}}\left(1+\psi_{j}\left(\theta^{\omega_{1}}, \ldots, \theta^{\omega_{s}}\right)\right)
$$

avec $\omega_{0, j}, \omega_{1}, \ldots, \omega_{s} \in \mathbb{Z}^{p} ; \theta^{\omega_{0, j}}, \theta^{\omega_{1}}, \ldots, \theta^{\omega_{s}} \in \Theta ; \psi_{j}$ analytique et $\psi_{j}(0)=0$, on a pour certaines déterminations des $a_{j}^{1 / m}$,

$$
\psi\left(\ldots, a_{j}^{1 / m} \theta^{\omega_{0, j / m}}\left(1+\psi_{j}\left(\theta^{\omega_{1}}, \ldots, \theta^{\omega_{s}}\right)\right)^{1 / m}, \ldots\right)
$$

qui est racine de $Q(t, W)$, ce qui achève la démonstration.

1.8. Exemple. Si $(\alpha)=\left(\alpha_{1}, \ldots, \alpha_{p}\right)$ est une suite de réels $>0$, on note $\mathcal{A}^{(\alpha)}$ l'algèbre de tous les germes en $0 \in \mathbb{R}^{+}$de fonctions $\varphi\left(t^{\alpha_{1}}, \ldots, t^{\alpha_{p}}\right)$ avec $\varphi \in \mathbb{C}\left\{y_{1}, \ldots, y_{p}\right\}$ et l'on pose $\mathcal{A}=\bigcup_{(\alpha)} \mathcal{A}^{(\alpha)}$ ( $p$ variable). D'après le théorème $1.4, \mathcal{K}_{\mathbb{C}}$ est algébriquement clos; la suite $\left(\alpha_{1}, \ldots, \alpha_{p}\right)$ étant fixée, il en est de même du corps des fractions de $\underline{\mathcal{A}}_{\mathbb{C}}^{(\alpha)}$ où $\underline{\mathcal{A}}^{(\alpha)}=\bigcup_{(\beta)} \mathcal{A}^{(\beta)},(\beta)$ décrivant l'ensemble de toutes les suites $\left(\beta_{1}, \ldots, \beta_{q}\right)$ de réels $>0$ tels que $\forall i, \beta_{i} \in \mathbb{Q} \alpha_{1}+\ldots+\mathbb{Q} \alpha_{p}$; item pour le corps des fractions de $\overline{\mathcal{A}}_{\mathbb{C}}^{(\alpha)}$ où $\overline{\mathcal{A}}^{(\alpha)}=\bigcup_{(\beta)} \mathcal{A}^{(\beta)},(\beta)$ décrivant l'ensemble de toutes les suites $\left(\beta_{1}, \ldots, \beta_{q}\right)$ de réels $>0$ tels que $\forall i, \beta_{i} \in \mathbb{Q}\left(\alpha_{1}, \ldots, \alpha_{p}\right)$.

On note $\mathcal{A}_{n, m}^{(\alpha)}$ l'algèbre des germes de fonctions à l'origine de $\left(\mathbb{R}^{+}\right)^{n} \times \mathbb{R}^{m}$ qui s'écrivent sous la forme

$$
\varphi\left(x_{1}^{\alpha_{1}}, \ldots, x_{1}^{\alpha_{p}} ; x_{2}^{\alpha_{1}}, \ldots, x_{2}^{\alpha_{p}} ; \ldots ; x_{n}^{\alpha_{1}}, \ldots, x_{n}^{\alpha_{p}} ; y_{1}, \ldots, y_{m}\right)
$$

( $\varphi$ est analytique à l'origine de $\mathbb{R}^{p n+m}$ à valeurs réelles; $x=\left(x_{1}, \ldots, x_{n}\right)$ paramétrise $\left(\mathbb{R}^{+}\right)^{n}$ et $y=\left(y_{1}, \ldots, y_{m}\right)$ paramétrise $\left.\mathbb{R}^{m}\right)$. On pose $\mathcal{A}_{n, m}=\bigcup_{(\alpha)} \mathcal{A}_{n, m}^{(\alpha)}$ et on note $\overline{\mathcal{A}}_{n, m}^{(\alpha)}$ l'algèbre des germes $f:\left(\left(\mathbb{R}^{+}\right)^{n} \times \mathbb{R}^{m}, 0\right) \rightarrow \mathbb{R}$ qui s'écrivent $f=f_{0} \circ f_{1} \circ \ldots \circ f_{s}$; $f_{k}:\left(\left(\mathbb{R}^{+}\right)^{n_{k}} \times \mathbb{R}^{m_{k}}, 0\right) \rightarrow\left(\left(\mathbb{R}^{+}\right)^{n_{k-1}} \times \mathbb{R}^{m_{k-1}}, 0\right)$ ayant ses composantes dans $\mathcal{A}_{n_{k}, m_{k}}^{(\alpha)} ;$ $\overline{\mathcal{A}}_{n, m}^{(\alpha)}$ est donc la plus petite algèbre qui est stable par composition et qui contient $\mathcal{A}_{n, m}^{(\alpha)}$; enfin on pose $\overline{\mathcal{A}}_{n, m}=\bigcup_{(\alpha)} \overline{\mathcal{A}}_{n, m}^{(\alpha)}$.

2. Etude des semi-analytiques définis à l'aide de $\overline{\mathcal{A}}_{n, m}$. Soit $f_{i j}$ un nombre fini d'éléments de $\overline{\mathcal{A}}_{n, m}, f_{i j}(0)=0$; on considère le germe d'ensemble semi-analytique $X=\bigcup_{i} \bigcap_{j}\left\{(x, y) \in\left(\mathbb{R}^{+}\right)^{n} \times \mathbb{R}^{m}: f_{i j}(x, y) ? 0\right\}$ où ? signifie $=$ ou $>$. Un tel germe $X$ 
admet localement un nombre fini de composantes connexes; cela est une conséquence de la théorie de Khovanskiur, mais nous allons retrouver ce résultat et certains autres que la théorie de Khovanskiǔ ne permet pas d'obtenir, en utilisant une méthode déjà employée dans [5]; on comparera aussi ces résultats avec ceux de [7], mais la famille de fonctions envisagée ici est beaucoup plus particulière. On démontre en particulier le lemme du petit chemin suivant :

2.1. ThÉORÈme. Avec les notations précédentes, si $X \not \subset\{0\}$, il existe un arc $\xi$ : $\left[0, \varepsilon\left[\ni t \rightarrow(x(t) ; y(t)) \in\left(\mathbb{R}^{+}\right)^{n} \times \mathbb{R}^{m}\right.\right.$ tel que $\xi(0)=0$ et $\xi(] 0, \varepsilon[) \subset X \backslash\{0\}$, à composantes dans $\mathcal{A} ;$ plus précisément, si les $f_{i j}$ sont dans $\overline{\mathcal{A}}_{n, m}^{(\alpha)}$, les composantes de $\xi$ sont dans $\overline{\mathcal{A}}^{(\alpha)}$.

2.2. Avant de démontrer 2.1, faisons deux remarques préliminaires :

2.2.1. Pour la démonstration, on peut remplacer $\overline{\mathcal{A}}_{n, m}$ par $\mathcal{A}_{n, m}$; en effet, on peut supposer que chaque $f_{i j} \in \overline{\mathcal{A}}_{n, m}$ s'écrit $f_{i, j}=f_{i j, 0} \circ \ldots \circ f_{i j, s}$ avec pour $k=1, \ldots, s$ $f_{i j, k}:\left(\left(\mathbb{R}^{+}\right)^{n_{k}} \times \mathbb{R}^{m_{k}}, 0\right) \rightarrow\left(\left(\mathbb{R}^{+}\right)^{n_{k-1}} \times \mathbb{R}^{m_{k-1}}, 0\right)$ et $f_{i j, k}$ a ses composantes dans $\mathcal{A}_{n_{k}, m_{k}}$. Soit $\pi$ la projection canonique de $\bigoplus_{k=0}^{s}\left(\mathbb{R}^{+}\right)^{n_{k}} \times \mathbb{R}^{m_{k}} \operatorname{sur}\left(\mathbb{R}^{+}\right)^{n_{s}} \times \mathbb{R}^{m_{s}}=\left(\mathbb{R}^{+}\right)^{n} \times \mathbb{R}^{m}$; en utilisant les graphes des $f_{i j, k}$, on voit que $X=\pi\left(X^{\prime}\right)$ où $X^{\prime}$ est un semi-analytique défini à l'aide de $\mathcal{A}_{n^{\prime}, m^{\prime}}$ avec $n^{\prime}=\sum_{k=0}^{s} n_{k}, m^{\prime}=\sum_{k=0}^{s} m_{k}^{\prime}$. Le résultat pour $X^{\prime}$ entraîne évidemment le résultat pour $X$.

2.2.2. Si $f \in \overline{\mathcal{A}}_{n, m}$ on a $|f| \in \overline{\mathcal{A}}_{n, m}$ car $|f|=\left(f^{2}\right)^{1 / 2}$; le germe $f \geq 0$ est donc le germe analytique $|f|-f=0$; les germes semi-analytiques ne sont donc rien d'autre que les germes constructibles.

2.3. On va utiliser des éclatements très simples que nous allons préciser; considérons l'éclatement $\pi: \widetilde{\mathbb{R}}^{n} \rightarrow \mathbb{R}^{n}$ d'un hyperplan de coordonnées de codimension deux de $\mathbb{R}^{n}$ $(n \geq 2)$, par exemple l'hyperplan $x_{1}=x_{2}=0$; la fibre $\pi^{-1}(0)$ s'identifie à l'espace projectif $\mathbb{P}_{1}(\mathbb{R})$ paramétré par les coordonnées homogènes $x_{1} \circ \pi, x_{2} \circ \pi$; dans cette fibre, on distingue les deux points $\dot{x}_{1}, \dot{x}_{2}$ (nous les appellerons singuliers) corespondant aux axes de coordonnées $x_{1}, x_{2}$; si on choisit par exemple le point $a=\dot{x}_{1}$, on a un système canonique de coordonnées locales en $a$ pour $\widetilde{\mathbb{R}}^{n}$, noté $\left(u_{1}, \ldots, u_{n}\right)$, tel que $x_{1}=u_{1}$, $x_{2}=u_{1} u_{2}, x_{3}=u_{3}, \ldots, x_{n}=u_{n}$. L'image réciproque par $\pi_{a}$ du quadrant $\left(\mathbb{R}^{+*}\right)^{n}$ est le quadrant $\left(\mathbb{R}^{+*}\right)^{n}:\left\{u_{i}>0,1 \leq i \leq n\right\}$. Les autres points de la fibre sont dits réguliers; en un tel point $a \in \pi^{-1}(0)$, on a un système de coordonnées locales $\left(u_{1}, \ldots, u_{n}\right)$ tel que $x_{1}=u_{2}\left(\lambda+u_{1}\right) ; x_{2}=u_{2} ; x_{3}=u_{3}, \ldots, x_{n}=u_{n}$, avec $\lambda \neq 0$. L'image réciproque par $\pi_{a}$ du quadrant $\left(\mathbb{R}^{+*}\right)^{n}$ est $\mathbb{R} \times\left(\mathbb{R}^{+*}\right)^{n-1}$ si $\lambda>0$; si $\lambda<0$ elle est vide. Visiblement, si $f \in \mathcal{A}_{n, m}$ on a $f \circ\left(\pi_{a} \times 1_{\mathbb{R}^{m}}\right) \in \mathcal{A}_{n, m}$ si $a$ est singulier; si $a$ est régulier, $\lambda>0$, on a $f \circ\left(\pi_{a} \times 1_{\mathbb{R}^{m}}\right) \in \mathcal{A}_{n-1, m+1}$.

Appelons transformation élémentaire en $0 \in\left(\mathbb{R}^{+}\right)^{n}$ le composé $\theta \circ \pi:\left(\pi^{-1}\left(\mathbb{R}^{+}\right)^{n}\right.$, $\left.\pi^{-1}(0)\right) \rightarrow\left(\left(\mathbb{R}^{+}\right)^{n}, 0\right)$ de l'éclatement $\pi$ d'un $(n-2)$-plan de coordonnées et d'un homéomorphisme $\theta: \theta\left(x_{1}, \ldots, x_{n}\right)=\left(x_{1}^{\beta_{1}}, \ldots, x_{n}^{\beta_{n}}\right)$ avec $\beta_{i}$ réels $>0$. On peut appliquer une transformation élémentaire en $0 \in\left(\mathbb{R}^{+}\right)^{n}$, puis recommencer en chacun des deux points singuliers de $\pi$, et l'on peut itérer un nombre fini de fois ce processus. On obtient ainsi 
un "arbre fini de transformations élémentaires" :

$$
\begin{aligned}
& \ldots \rightrightarrows \rightarrow \\
& \ldots \rightrightarrows \rightarrow
\end{aligned} \rightarrow
$$

La remarque suivante est essentielle pour la suite :

2.4. LEMME. Soit $\Omega$ un sous-ensemble fini de $\left(\mathbb{R}^{+}\right)^{n} ;$ il existe un arbre fini de transformations élémentaires tel que si a est l'un des deux points singuliers d'une flèche initiale de l'arbre et si $\pi:\left(\left(\mathbb{R}^{+}\right)^{n}, 0\right) \ni\left(y_{1}, \ldots, y_{n}\right) \rightarrow \pi\left(y_{1}, \ldots, y_{n}\right) \in\left(\left(\mathbb{R}^{+}\right)^{n}, 0\right)$ est le morphisme composé des transformations élémentaires entre a et 0 , on ait $\forall \omega \in \Omega, \pi^{*}\left(x^{\omega}\right)=y^{\mu}$, les $\mu$ formant un sous-ensemble de $\left(\mathbb{R}^{+}\right)^{n}$ totalement ordonné pour l'ordre produit.

Preuve. Evidemment, $\pi^{*}$ est injective et $\pi^{*}$ conserve l'ordre sur les monômes; on peut donc supposer card $\Omega=2$, car par itération on a le résultat pour un $\Omega$ quelconque. Si $x^{\omega}$ et $x^{\omega^{\prime}}$ sont deux monômes, on peut diviser par le facteur commun à $x^{\omega}$ et $x^{\omega^{\prime}}$ et supposer que $x^{\omega}=x_{1}^{\omega_{1}} \ldots x_{r}^{\omega_{r}}$ et $x^{\omega^{\prime}}=x_{s+1}^{\omega_{s+1}} \ldots x_{n}^{\omega_{n}}, s \geq r, \omega_{i}>0$, après une éventuelle permutation de coordonnées. On procède alors par récurrence descendante sur $r+(n-s)$; si $r=0$ ou $n-s=0$ (i.e. $x^{\omega}=1$ ou $x^{\omega^{\prime}}=1$ ), il n'y a rien à démontrer; sinon, on peut supposer en appliquant une transformation $\theta$ que tous les $\omega_{i}$ sont égaux à 1 ; en éclatant l'hyperplan $x_{1}=x_{s+1}=0$, on obtient alors les monômes $x_{1} \ldots x_{r}$ et $x_{1} x_{s+1} \ldots x_{n}$ (ou $x_{1} \ldots x_{r} x_{s+1}$ et $\left.x_{s+1} \ldots x_{n}\right)$; divisant par $x_{1}$ dans le premier cas et par $x_{s+1}$ dans le second, on peut appliquer l'hypothèse d'induction.

2.5. Lemme. Supposons que le théorème 2.1 est vrai pour $\mathcal{A}_{n, m-1}$ et supposons que le semi-analytique $X$ considéré en 2.1 est défini par des $f_{i j}$ qui sont des polynômes distingués en $y_{m}$ à coefficients dans $\mathcal{A}_{n, m-1}\left(\mathbb{R}^{m-1}\right.$ est paramétré par $\left.y^{\prime}=\left(y_{1}, \ldots, y_{m-1}\right)\right)$; alors le théorème 2.1 est vrai pour $X$.

Preuve. Posons $f=\prod_{i, j} f_{i j}=\sum_{k=1}^{M} a_{k}\left(x ; y^{\prime}\right) y_{m}^{k-1}$ et considérons le polynôme générique $F=\sum_{k} a_{k} y_{m}^{k-1} \in \mathbb{R}\left[a ; y_{m}\right]$ où $a$ désigne le paquet de variable $a_{k}$ et décrit un $\mathbb{R}^{M}$. Soit $\theta$ l'application $\left(\left(\mathbb{R}^{+}\right)^{n} \times \mathbb{R}^{m-1}, 0\right) \ni\left(x ; y^{\prime}\right) \rightarrow\left(a_{k}\left(x, y^{\prime}\right)\right) \in\left(\mathbb{R}^{M}, 0\right)$.

Comme conséquence du théorème de Tarski-Seidenberg (cf. [1], "méthode du saucissonnage"), il existe une partition fini de $\mathbb{R}^{M}$ en semi-algébriques connexes $W_{\alpha}$ tels que au-dessus de chaque $W_{\alpha}, F$ ait un nombre constant $l_{\alpha}$ de racines réelles :

$$
\mathcal{Y}_{\alpha}^{(1)}(a)<\mathcal{Y}_{\alpha}^{(2)}(a)<\ldots<\mathcal{Y}_{\alpha}^{\left(l_{\alpha}\right)}(a)
$$

Il en résulte que $X \cap\left(\theta^{-1}\left(W_{\alpha}\right) \times \mathbb{R}\right)$ est une union disjointe de "tranches" de la forme :

$$
\begin{aligned}
T_{l} & =\left\{\left(x, y^{\prime}, y_{m}\right):\left(x, y^{\prime}\right) \in \theta^{-1}\left(W_{\alpha}\right) \text { et } y_{m}=\mathcal{Y}_{\alpha}^{(l)}\left(\theta\left(x, y^{\prime}\right)\right)\right\}, \\
T_{l, l+1} & =\left\{\left(x, y^{\prime}, y_{m}\right):\left(x, y^{\prime}\right) \in \theta^{-1}\left(W_{\alpha}\right) \text { et } \mathcal{Y}_{\alpha}^{(l)}\left(\theta\left(x, y^{\prime}\right)\right)<y_{m}<\mathcal{Y}_{\alpha}^{(l+1)}\left(\theta\left(x, y^{\prime}\right)\right)\right\}, \\
T_{-\infty, 1} & =\left\{\left(x, y^{\prime}, y_{m}\right):\left(x, y^{\prime}\right) \in \theta^{-1}\left(W_{\alpha}\right) \text { et } y_{m}<\mathcal{Y}_{\alpha}^{(1)}\left(\theta\left(x, y^{\prime}\right)\right)\right\}, \\
T_{l_{\alpha}, \infty} & =\left\{\left(x, y^{\prime}, y_{m}\right):\left(x, y^{\prime}\right) \in \theta^{-1}\left(W_{\alpha}\right) \text { et } \mathcal{Y}_{\alpha}^{\left(l_{\alpha}\right)}\left(\theta\left(x, y^{\prime}\right)\right)<y_{m}\right\} .
\end{aligned}
$$

Si $X$ est contenu dans la droite des $y_{m}\left(x=y^{\prime}=0\right)$, le résultat est évident. Sinon, il existe un $\alpha$ tel que $0 \notin \theta^{-1}\left(W_{\alpha}\right) ; 0 \in \overline{\theta^{-1}\left(W_{\alpha}\right)} ; X$ contient une tranche $T$ au-dessus de $\theta^{-1}\left(W_{\alpha}\right)$. Par hypothèse, il existe un arc $\xi^{\prime}:\left(\left[0, \varepsilon[, 0) \rightarrow\left(\left(\mathbb{R}^{+}\right)^{n} \times \mathbb{R}^{m-1}, 0\right)\right.\right.$ à coefficients dans $\mathcal{A}$, tel que $\xi^{\prime}(] 0, \varepsilon[) \subset \theta^{-1}\left(W_{\alpha}\right)$. Supposons que $T$ est de la forme $T_{l} ; \xi_{m}=\mathcal{Y}_{\alpha}^{(l)}\left(\theta\left(\xi^{\prime}(t)\right)\right)$ est 
alors racine du polynôme distingué en $y_{m} f\left(\xi^{\prime}(t) ; y_{m}\right)$ et donc cette racine appartient à $\mathcal{A}$ d'après le lemme de Puiseux 1.4. L'application $\xi^{\prime}$ se relève donc en un arc $\xi=\left(\xi^{\prime}, \xi_{m}\right)$ tel que $\xi(] 0, \varepsilon[) \subset T_{l} \subset X \backslash\{0\}$. Si $T=T_{l, l+1}$, on choisit par exemple $\xi_{m}=\frac{1}{2}\left(\mathcal{Y}_{\alpha}^{(l)}\left(\theta\left(\xi^{\prime}(t)\right)\right)+\right.$ $\left.\mathcal{Y}_{\alpha}^{(l+1)}\left(\theta\left(\xi^{\prime}(t)\right)\right)\right)$; si $T=T_{-\infty, 1}$, on peut choisir $\xi_{m}=\mathcal{Y}_{\alpha}^{1}\left(\theta\left(\xi^{\prime}(t)\right)\right)-t$; si $T=T_{l_{\alpha}, \infty}$, $\xi_{m}=\mathcal{Y}_{\alpha}^{\left(l_{\alpha}\right)}\left(\theta\left(\xi^{\prime}(t)\right)\right)+t$.

2.6. Preuve du théorème 2.1. On peut supposer d'après 2.2 .1 que les $f_{i j}$ sont dans $\mathcal{A}_{n, m}$; on procède par induction sur le couple $(n, n+m)$ ordonné lexicographiquement (si $n=m=0$, il n'y a rien à dire). On peut admettre que $X \cap\left(\left(\mathbb{R}^{+*}\right)^{n} \times \mathbb{R}^{m}\right)$ est non vide, car sinon, on se ramène à un $n^{\prime}<n$, et l'on peut supposer que $X \subset\left(\mathbb{R}^{+*}\right)^{n} \times \mathbb{R}^{m}$.

Ecrivons chaque $f_{i j}$ sous la forme $f_{i j}=\sum_{\omega \in \Omega_{i j}} x^{\omega}\left(f_{i j, \omega}(y)+g_{i j, \omega}(x, y)\right)$ où $\Omega_{i j}$ est un sous-ensemble fini de $\left(\mathbb{R}^{+}\right)^{n} ; f_{i j, \omega} \in \mathbb{R}\left\{y_{1}, \ldots, y_{m}\right\}$ et $f_{i j, \omega} \neq 0 ; g_{i j, \omega} \in \mathcal{A}_{n, m}$ et $g_{i j, \omega}(0, y)=0$.

Posons $\Omega=\bigcup_{i, j} \Omega_{i j}$ et appliquons le lemme 2.6 à $\Omega$ si $n \geq 2$; il existe un arbre de transformations élémentaires vérifiant les conclusions de 2.4 ; si $a$ est un point de la fibre au-dessus 0 , notons $\pi_{a}$ le morphisme composé des transformations entre $a$ et 0 et soit $\underline{\pi}_{a}:\left(\left(\mathbb{R}^{+}\right)^{n} \times \mathbb{R}^{m}, 0\right) \rightarrow\left(\left(\mathbb{R}^{+}\right)^{n} \times \mathbb{R}^{m}, 0\right)$ le morphisme $\pi_{a} \times$ identité. Il suffit de montrer que $\forall a$, le théorème est vrai pour le semi-analytique supposé non vide

$$
X_{a}=\underline{\pi}_{a}^{-1}(X)=\bigcup_{i}\left\{(x, y): f_{i j} \circ \underline{\pi}_{a}(x, y) ? 0\right\} .
$$

Distinguons deux cas (on suppose $n \geq 2$ ) :

- $a$ est un point singulier d'une flèche initiale de l'arbre; d'après le lemme 2.4, chaque $f_{i j} \circ \underline{\pi}_{a}$ s'écrit $x^{\mu_{i j}}\left(h_{i j}(y)+g_{i j}(x, y)\right)$ avec $\mu_{i j} \in\left(\mathbb{R}^{+}\right)^{n} ; h_{i j} \in \mathbb{R}\left\{y_{1}, \ldots, y_{m}\right\}$ et $h_{i j} \neq 0$; $g_{i j} \in \mathcal{A}_{n, m}$ et $g_{i j}(0, y)=0$.

Distinguons encore deux cas :

* Si $m=0$, alors $h_{i j}$ est une constante réelle non nulle; le germe semi-analytique $X_{a}$ est donc de la forme

$$
\bigcup_{i} \bigcap_{j}\left\{x \in\left(\mathbb{R}^{+*}\right)^{n}: x^{\mu_{i j}} ? 0\right\}
$$

et le théorème est trivialement vrai pour $X_{a}\left(X_{a}=\emptyset\right.$ ou $\left.X_{a}=\left(\mathbb{R}^{+*}\right)^{n}\right)$.

$*$ Si $m>0$ et si $\varphi_{i j}(x, y)=h_{i j}(y)+g_{i j}(x, y)$, on a

$$
X_{a}=\bigcup_{i} \bigcap_{j}\left\{(x, y) \in\left(\mathbb{R}^{+*}\right)^{n} \times \mathbb{R}^{m}: \varphi_{i j}(x, y) ? 0\right\} .
$$

Mais $\varphi_{i j}(0, y) \neq 0$ et d'après le théorème de préparation analytique usuel, après un éventuel changement linéaire de coordonnées sur les $y$, on peut supposer que chaque $\varphi_{i j}$ est équivalent dans $\mathcal{A}_{n, m}$ à un polyôme distingué $\psi_{i j} \in \mathcal{A}_{n, m-1}\left[y_{m}\right]$. D'après l'hypothèse d'induction, le théorème est vrai pour $\mathcal{A}_{n, m-1}$ et donc il est vrai pour $X_{a}$ d'après le lemme 2.5 .

- $a$ est un point régulier d'une fléche initiale de l'arbre; mais alors les $f_{i j} \circ \underline{\pi}_{a}$ appartiennent à $\mathcal{A}_{n-1, m+1}$ et on applique l'hypothèse d'induction.

Reste enfin à examiner le cas $n=0$ ou $n=1$; dans le second cas, on peut écrire 
$f_{i j}(x, y)=x^{\alpha} g_{i j}(x, y)$ avec $g_{i j}(0, y) \neq 0$; on peut là encore appliquer le théorème de préparation à $g_{i j}(x, y)$ après un éventuel changement linéaire de coordonnées sur les $y$; le théorème résulte alors du lemme 2.5 et de l'hypothèse d'induction. Si $n=0$, c'est le cas analytique bien connu, et la démonstration est analogue.

Enfin, on vérifie facilement que si les $f_{i j}$ sont dans $\mathcal{A}_{n, m}^{(\alpha)}$, alors les composantes de $\xi$ sont dans $\overline{\mathcal{A}}^{(\alpha)}$.

2.7. Remarque. La méthode précédente permet de démontrer beacoup plus. D'abord, une fonction de $\overline{\mathcal{A}}_{n, m}$ vérifie l'inégalité de Łojasiewicz par rapport au germe de ses zéros (ce résultat est un cas particulier d'une situation beaucoup plus générale, cf. [4] ou [6]).

Ensuite, si $X$ est un "germe semi-analytique" défini à l'aide d'éléments de $\mathcal{A}_{n, m}$, il existe un nombre fini d'applications $\pi_{i}:\left(\left(\mathbb{R}^{+}\right)^{n}, 0\right) \rightarrow\left(\left(\mathbb{R}^{+}\right)^{n}, 0\right)$, chaque $\pi_{i}$ étant composé d'un nombre fini de transformations élémentaires (cf. 2.3), et pour chaque $i$, un représentant $\underset{\sim}{X}{ }_{i}$ d'un "germe semi-analytique" défini aussi à l'aide d'éléments de $\mathcal{A}_{n, m}$ tel que

$$
\underset{\sim}{X}=\bigcup_{i}\left(\pi_{i} \times 1_{\mathbb{R}^{m}}\right)\left(\underset{\sim}{X_{i}}\right)
$$

( $X$ est un représentant convenable de $X$ ). On a un résultat analogue quand on remplace l'adhérence $\underset{\sim}{X}$ par une composante connexe de $\underset{\sim}{X}$. On démontre aussi que le nombre de ces composantes connexes est fini (mais ceci est une conséquence de la théorie de Khovanskiı̌). Ainsi, l'adhérence ou une composante connexe d'un "semi-analytique" ne sont peut être pas "semi-analytiques", mais quitte à effectuer un nombre fini de transformations élémentaires, cela est vrai.

\subsection{Remarques}

2.8.1. On peut se demander si le théorème 2.1 reste vrai dans le cas "algébrique", i.e. lorsque les germes qui définissent $X$ sont des fonctions de Nash en un nombre fini de monômes $x^{\omega} y^{\mu}, \omega \in\left(\mathbb{R}^{+}\right)^{n}, \mu \in \mathbb{N}^{n}$. Bien entendu, on exige que la courbe $\xi$ soit aussi "algébrique". Sous cette forme cela n'est pas vrai; par exemple, pour résoudre en $x$ l'équation $y=x\left(1+x^{\alpha}\right), \alpha$ irrationnel $>0$, on pose $x=y(1+u)$ et l'on obtient l'équation $u+y^{\alpha}(1+u)^{\alpha}+u y^{\alpha}(1+u)^{\alpha}=0$ qui admet d'après le théorème des fonctions implicites, une solution $u=\varphi\left(y^{\alpha}\right)$ avec $\varphi$ analytique, mais pas de Nash. Il faut donc redéfinir ce que l'on appelle "courbe algébrique".

2.8.2. Soit $\varphi(u, v) \in \mathbb{R}\left[u^{\alpha_{1}}, \ldots, u^{\alpha_{p}} ; v^{\alpha_{1}}, \ldots, v^{\alpha_{p}}\right]$ avec $\alpha_{i}>0$, et soit $\mu>0, \mu$ irrationnel; alors $\varphi\left(1-y, 1-y^{\mu}\right)=0 \Rightarrow \varphi=0$; en effet, on peut écrire $\varphi(u, v)=$ $\sum v^{\beta_{i}} \varphi_{i}(u)$ où $\beta_{1}<\beta_{2}<\ldots$ est une suite strictement croissante finie de réels positifs, et on montre par induction sur $i$ que $\varphi_{i}=0$. Par exemple, quitte à diviser par $v^{\beta_{1}}$, on peut supposer $\beta_{1}=0$ et l'on a $\varphi_{1}(1-y)=0$, donc $\varphi_{1}=0$, car en prolongeant au complexe et en tournant autour de 0 sans tourner autour de 1 , on voit qu'une détermination holomorphe de $\varphi_{1}(1-y)$ admettrait comme zéros tous les points $e^{2 k \pi i / \mu}, k \in \mathbb{Z}$.

Considérons alors l'application "algébrique", inspirée de celle d'Osgood :

$$
\left(\left(\mathbb{R}^{+}\right)^{2}, 0\right) \ni(x, y) \stackrel{f}{\longrightarrow}\left(x, x(1-y), x\left(1-y^{\mu}\right)\right) \in\left(\left(\mathbb{R}^{+}\right)^{3}, 0\right) .
$$


Si $\psi \in \mathcal{A}_{3,0}$ et $\psi \circ f=0$, on a $\psi=0$; en effet, si $\psi(X, Y, Z)=\sum_{\alpha, \beta, \gamma} a_{\alpha \beta \gamma} X^{\alpha} Y^{\beta} Z^{\gamma}$, on a

$$
\psi\left(x, x(1-y), x\left(1-y^{\mu}\right)\right)=\sum_{\delta} x^{\delta}\left(\sum_{\alpha+\beta+\gamma=\delta} a_{\alpha \beta \gamma}(1-y)^{\beta}\left(1-y^{\mu}\right)^{\gamma}\right)=0
$$

chacune de ces parenthèses est nulle et d'après la remarque précédente, $\psi=0$. Il est donc vain d'espérer un résultat genre Tarski-Seidenberg et la projection d'un "semi-algébrique" n'est même pas "semi-analytique".

\section{Exemples de paramétrisations en dimension deux}

3.1. On note $\Theta$ la famille de toutes les fonctions

$$
\theta_{\alpha}(t)=(1 / t)^{\alpha_{0}}(\log 1 / t)^{\alpha_{1}} \ldots\left(\log _{q} 1 / t\right)^{\alpha_{q}},
$$

où les $\alpha_{i}$ sont des réels tels que $\theta_{\alpha}(t) \rightarrow 0$ quand $t \rightarrow 0$ (donc les $\alpha_{i}$ ne sont pas tous nuls et le premier $\alpha_{i}$ non nul est $\left.<0\right)$. La famille $\Theta$ vérifie les conditions de 1.2.4; on note $\mathcal{B}$ l'algèbre $\mathcal{H}_{\Theta}$ associée à $\Theta$ (cf. 1.2.4) et plus généralement on note $\mathcal{B}_{n}$ l'algèbre des germes de fonctions à l'origine de $\left(\mathbb{R}^{+}\right)^{n}$ de la forme

$$
f\left(\theta_{\alpha^{1}}\left(x_{1}\right), \ldots, \theta_{\alpha^{p}}\left(x_{1}\right) ; \ldots ; \theta_{\alpha^{1}}\left(x_{n}\right), \ldots, \theta_{\alpha^{p}}\left(x_{n}\right)\right)
$$

avec $\theta_{\alpha^{i}}(t) \in \Theta$ ( $p$ et les $\alpha^{i}$ dépendent de $f$ et $f$ est analytique à valeurs réelles).

Soient $\theta_{\alpha}, \theta_{\beta} \in \Theta, \alpha=\left(\alpha_{0}, \ldots, \alpha_{q}\right), \beta=\left(\beta_{0}, \ldots, \beta_{q}\right), \lambda>0$; supposons $\alpha_{0} \neq 0$, donc $\alpha_{0}<0$. On vérifie facilement que

$$
\theta_{\beta} \circ\left(\lambda \theta_{\alpha}\right)=\lambda^{-\beta_{0}}\left(-\alpha_{0}\right)^{\beta_{1}} \cdot \theta_{\gamma} \cdot(1+\varphi)
$$

avec $\varphi \in \mathcal{B}, \varphi(0)=0 ; \gamma_{0}=-\alpha_{0} \beta_{0}$ et $\gamma_{i}=-\alpha_{i} \beta_{0}+\beta_{i}$, si $i>0$. En particulier, si $\beta_{0}=1 / \alpha_{0}$ et $\beta_{i}=\alpha_{i} / \alpha_{0}, i>0, \theta_{\beta} \circ\left(\lambda \theta_{\alpha}\right)(t)$ est équivalent dans $\mathcal{B}$ à $\lambda^{-1 / \alpha_{0}}\left(-\alpha_{0}\right)^{\alpha_{1} / \alpha_{0}} t$. On en déduit aussi facilement que $\mathcal{B}$ est stable pour la composition des applications.

Remarquons enfin que si $f$ et $g$ sont deux germes de fonctions réelles positives, non oscillantes, à l'origine de $\mathbb{R}^{+}, f \sim g \Rightarrow \theta \circ f \sim \theta \circ g, \forall \theta \in \Theta$ (on rappelle que $f \sim g$ signifie que $g(x) / f(x)$ tend vers une limite finie non nulle quand $x \rightarrow 0, x>0)$.

3.2. Lemme. Si $\varphi \in \mathcal{B}, \varphi(t+t y)$ appartient à l'algèbre notée $\mathcal{B}\{y\}$ formée des germes qui sont analytiques réels en y et en un nombre fini de $\theta \in \Theta$.

Preuve. On a

$\log (t+t y)^{-1}=\log \frac{1}{t}-\log (1+y) ; \quad \log _{2}(t+t y)^{-1}=\log _{2} \frac{1}{t}+\log \left(1-\frac{\log (1+y)}{\log 1 / t}\right) ; \ldots$ et donc

$$
\log _{q}(t+t y)^{-1}=\log _{q} \frac{1}{t}+\varphi\left(\left(\log \frac{1}{t}\right)^{-1}, \ldots,\left(\log _{q-1} \frac{1}{t}\right)^{-1}, y\right)
$$

avec $\varphi$ analytique réelle et $\varphi(0)=0$. On a donc

$$
\theta_{\alpha}(t+t y)=\theta_{\alpha}(t) \psi\left(\left(\log \frac{1}{t}\right)^{-1}, \ldots,\left(\log _{q-1} \frac{1}{t}\right)^{-1}, y\right)
$$

avec $\psi$ analytique réelle et $\psi(0)=1$; d'où le résultat.

3.3. Soit $f(x, y) \in \mathcal{B}_{2}, f(0,0)=0$; d'après la théorie de Khovanskiư, la courbe $f(x, y)=0$ au voisinage de 0 dans $\left(\mathbb{R}^{+}\right)^{2}$ est formée (outre peut-être les demi-axes positifs) 
d'un nombre fini de branches $y=\varphi(x)(\varphi \neq 0$ et $\varphi(0)=0)$ qui sont non oscillants, avec une tangente en 0 . Soit $\varphi$ l'une de ces branches et posons $f(x, y)=\sum a_{\alpha, \beta} \theta_{\alpha}(y) \theta_{\beta}(x)$, d'où $\sum a_{\alpha, \beta} \theta_{\alpha}(\varphi(x)) \theta_{\beta}(x)=0$. Considérons parmi tous les termes qui interviennent dans cette somme, ceux de multiplicité minimale; il y en a un nombre fini et il en existe au moins deux; ainsi, on a deux multi-indices $\left(\alpha^{\prime}, \beta^{\prime}\right) \neq\left(\alpha^{\prime \prime}, \beta^{\prime \prime}\right)$ tels que $\theta_{\alpha^{\prime}}(\varphi(x)) \theta_{\beta^{\prime}}(x) \sim$ $\theta_{\alpha^{\prime \prime}}(\varphi(x)) \theta_{\beta^{\prime \prime}}(x)$, d'où l'existence de deux multi-indices $\alpha$ et $\beta$ tels que $\theta_{\alpha}(\varphi(x)) \sim \theta_{\beta}(x)$. Posons

$$
\theta_{\alpha}(y)=\left(\frac{1}{y}\right)^{\alpha_{0}}\left(\log \frac{1}{y}\right)^{\alpha_{1}} \ldots\left(\log _{q} \frac{1}{y}\right)^{\alpha_{q}}
$$

et soit $\alpha_{r}$ le premier $\alpha_{i}$ non nul (donc $\alpha_{r}<0$ ). Faisons le changement de variables $u=$ $\log _{r} 1 / y$; en utilisant la formule d'inversion de 3.1 , on a une "équation caractéristique" :

$$
\log _{r} \frac{1}{\varphi(x)} \sim \theta_{\gamma}^{-1}(x), \quad \theta_{\gamma} \in \Theta
$$

Disons que cette équation caractéristique est d'ordre $r$ et remarquons que si $r \geq 1$ cette équation ne donne pas une équivalence pour $1 / \varphi(x)$ (si par exemple $\log u \sim v$, Log $u(x)=$ $A v(x)(1+\varepsilon(x))$ et $u(x)=e^{A v(x)} \cdot e^{A v(x) \varepsilon(x)}$, mais on ne sait pas contrôler le produit $v(x) \varepsilon(x)$ car $v(x) \rightarrow-\infty$ et $\varepsilon(x) \rightarrow 0$ quand $x \rightarrow 0, x>0)$.

Le cas $r=0$ est facile; c'est une situation générique qui est la seule à apparaître lorsque $f$ est une fonction analytique de certains $\theta_{\alpha}(x)$ et de $\theta_{\alpha^{1}}(y), \ldots, \theta_{\alpha^{p}}(y)$, les $\alpha_{0}^{1}, \ldots, \alpha_{0}^{p}$ étant linéairement indépendants sur $\mathbb{Q}$.

3.4. Proposition. Avec les notations précédentes, s'il existe une équation caractéristique (3.3.1) d'ordre 0 , on a $\varphi \in \mathcal{B}$.

Preuve. On a $\varphi(x)=A \theta_{\gamma}(x)(1+u(x))$ avec $u(x) \rightarrow 0$ quand $x \rightarrow 0$ et $A>0$; faisons le changement de variables $y=A \theta_{\gamma}(x)(1+u)$; d'après le lemme 3.2 et 3.1 ,

$$
f(x, y)=g(x, u) \in \mathcal{B}\{u\} .
$$

D'après le lemme 1.3 , quitte à mettre en facteur un élément de $\mathcal{B}=\mathcal{H}_{\Theta}$ on peut pour résoudre l'équation $g(x, u)=0$, supposer que $g(0, u) \neq 0$.

D'après le théorème de préparation analytique, on peut donc supposer que $g(x, u)$ est un polynôme distingué en $u$ à coefficients dans $\mathcal{B}$; la proposition résulte alors du lemme de Puiseux 1.4.

Je ne sais pas si l'on peut dire des choses analogues quand on remplace $\mathcal{B}_{2}$ par $\mathcal{B}_{n}$. Considérons un germe de courbe à l'origine de $\left(\mathbb{R}^{+}\right)^{n}$ défini par un système d'équations $f_{i}\left(x, y_{1}, \ldots, y_{n-1}\right)=0$, avec $f_{i} \in \mathcal{B}_{n}$, et considérons une branche de cette courbe $y_{i}=$ $\varphi_{i}(x), 1 \leq i \leq n-1$ (on suppose $\varphi_{i} \neq 0, \forall i$ ). Là encore par Khovanskiü les $\varphi_{i}(x)$ sont non oscillantes et l'on peut considérer des équations caractéristiques vérifiées par $\varphi_{1}, \ldots, \varphi_{n-1}$; malheureusement, ces équations caractéristiques ne déterminent pas les multiplicités de $\varphi_{1}, \ldots, \varphi_{n-1}$ en 0 . On a cependant le résultat suivant :

3.5. Proposition. Avec les notations précédentes, supposons que pour $i=1, \ldots$, $n-1$, on ait $\varphi_{i}(x) \sim \theta_{i}(x)$, avec $\theta_{i} \in \Theta ;$ alors $\forall i, \varphi_{i} \in \mathcal{B}$.

Preuve. On a $\varphi_{i}(x)=A_{i} \theta_{i}(x)\left(1+u_{i}(x)\right), u_{i}(x) \rightarrow 0$ quand $x \rightarrow 0$ et $A_{i}>0$; faisons le changement de variables $y_{i}=A_{i} \theta_{i}\left(1+u_{i}\right)$; d'après le lemme $3.2, f_{i}(x, y)=g_{i}(x, u) \in$ 
$\mathcal{B}\left\{u_{2}, \ldots, u_{n-1}\right\}$. Dans $\mathcal{B}\left\{u_{2}, \ldots, u_{n-1}\right\}$, on peut appliquer le théorème de préparation aux variables $u_{2}, \ldots, u_{n-1}$ et supposer, après un changement linéaire de coordonnées sur $u_{2}, \ldots, u_{n-1}$, que les $g_{i}$ sont des polynômes distingués en $u_{n-1}$. On procède alors par induction sur $n$ en utilisant les arguments du lemme 2.5 (essentiellement TarskiSeidenberg) et le lemme de Puiseux 1.4.

3.6. R e marque. Dans la proposition précédente, on utilise le fait que $\mathcal{B}$ est stable par composition. Si l'on ne suppose pas que les $\theta_{i}$ sont dans $\Theta$, le même changement de variables montre que les $\varphi_{i}(x)$ sont des fonctions analytiques en un nombre fini de fonctions qui, soit appartiennent à $\Theta$, soit sont de la forme $\theta_{i} \circ \theta$, avec $\theta \in \Theta$.

\subsection{EXEMPLES}

3.7.1. Soit $\varphi \in \mathcal{B}$ tel que $\varphi \sim \theta_{\alpha}$ avec $\alpha_{0}<0$; alors $\varphi$ est inversible dans $\mathcal{B}$ pour la composition des applications (on résout l'équation $x=\varphi(y)$ ). Par exemple, si $\varphi(y)=$ $y \log 1 / y$, on a

$$
y=\frac{x}{\log 1 / x}(1+u)
$$

d'où l'équation

$$
u+\left(-\frac{\log (1+u)}{\log 1 / x}+\frac{\log \log 1 / x}{\log 1 / x}\right)(1+u)=0 .
$$

En résolvant cette équation par le théorème des fonctions implicites, on trouve

$$
y=\frac{x}{\log 1 / x} \cdot \varphi\left(\frac{1}{\log 1 / x}, \frac{\log \log 1 / x}{\log 1 / x}\right)
$$

avec $\varphi \in \mathbb{R}\{u, v\}, \varphi(0,0)=1$.

On aurait un calcul analogue pour l'équation $x=y-y / \log y$; on peut résoudre cette équation en appliquant le théorème du point fixe à $y \mapsto x+y / \log y$, mais ceci est maladroit et le calcul est inextricable.

3.7.2. Soit $\varphi \in \mathbb{R}\{t\}, \varphi>0$ pour $t>0$; posons

$$
f_{\varphi}=\exp \left(-\frac{1}{x} \varphi\left(-\frac{1}{\log x}\right)\right) .
$$

$f_{\varphi}$ est un germe de fonction $C^{\infty}$ à l'origine de $\mathbb{R}^{+}$, plate à l'origine; en outre, $f_{\varphi} \sim f_{\varphi^{\prime}} \Leftrightarrow$ $\varphi=\varphi^{\prime}$. Les fonctions $f_{\varphi}$ ne peuvent pas être développées à l'aide de fonctions plus simples; en outre, $f_{\varphi}$ est solution de l'équation

$$
x+\frac{1}{\log y} \varphi\left(-\frac{1}{\log x}\right)=0,
$$

qui admet une équation caractéristique d'ordre 1. Cela montre que les fonctions élémentaires qui décrivent les solutions (lorsque les équations caractéristiques sont d'ordre $\geq 1$ ) doivent être choisies dans une classe très vaste de fonctions.

3.7.3. Soit à résoudre en $y$ l'équation

$$
x=y+\frac{1}{\log 1 / y}-\frac{1}{\log 1 / y \log _{2} 1 / y} .
$$


L'équation caractéristique est d'ordre 1 et l'on pose $Y=\frac{1}{\log 1 / y}$, d'où

$$
x=Y+\frac{Y}{\log Y}+e^{-1 / Y}
$$

On résout d'abord l'équation $Y+Y / \log Y=u$, d'où $Y=u(1+\varphi(-1 / \log u))$ avec $\varphi$ analytique, $\varphi(0)=0$; donc

$$
x=u+\exp (-1 / u(1+\varphi(-1 / \log u))) .
$$

On pose $u=x(1+x v)$; d'où

$$
v=f_{\varphi}(x)\left(1+v \theta\left(x, \frac{-1}{\log x}, v\right)\right)
$$

avec $\theta$ analytique; $f_{\varphi}=x^{-2} \exp (-1 / x(1+\varphi(-1 / \log x)))$; par le théorème des fonctions implicites,

$$
v=f_{\varphi}(x)\left(1+\theta^{\prime}\left(x, \frac{-1}{\log x}, f_{\varphi}(x)\right)\right)
$$

avec $\theta^{\prime}$ analytique, $\theta^{\prime}(0)=0$. On en déduit un développement de $u, Y$ et donc $y$ à l'aide de $f_{\varphi}$ et de fonctions de $\Theta$.

3.7.4. On a utilisé la remarque suivante : $\operatorname{si} u(x)=v(x)(1+v(x) \omega(x))$ avec $u, v, \omega \rightarrow 0$ quand $x \rightarrow 0, x>0$, on a $e^{-1 / u(x)}=e^{-1 / v(x)} \cdot e^{-\omega+v \omega^{2}-\ldots}$ et cette dernière exponentielle est analytique en $v$ et $\omega$.

3.8. Proposition. Avec les notations de 3.3, supposons $q=1$. Quitte à permuter $x$ et $y$, une branche de l'équation $f(x, y)=0$ admet toujours une paramétrisation de la forme $y=\varphi(x)$ avec $\varphi$ analytique en un nombre fini de $x^{\alpha}(\log 1 / x)^{\beta}\left(\log _{2} 1 / x\right)^{\gamma}$; ou de le forme $-1 / \log y=\varphi(x)$ avec $\varphi$ analytique en un nombre fini de $(\log 1 / x)^{\alpha} \exp \left(-\gamma(\log 1 / x)^{\beta}\right)$.

Preuve. Comme $q=1$, l'équation caractéristique s'écrit

$$
y^{\alpha}(\log 1 / y)^{\beta} \sim x^{\alpha^{\prime}}(\log 1 / x)^{\beta^{\prime}} .
$$

Si $\alpha>0$, on applique 3.4 et on résout en $y$; de même si $\alpha^{\prime}>0$, on peut résoudre en $x$. On peut donc supposer que l'équation caractéristique s'écrit

$$
\log 1 / y \sim(\log 1 / x)^{\mu-1}, \quad \mu>0
$$

et donc il existe $A>0$ tel que

$$
(\log 1 / y)^{\mu}=A^{-1}(\log 1 / x)(1+\varepsilon(x))
$$

où $\varepsilon(x) \rightarrow 0$ quand $x \rightarrow 0$. Posons $1 / v=\log 1 / y ; 1 / u=\left(A^{-1} \log 1 / x\right)^{\mu-1}$; donc $v=u\left(1+\varepsilon^{\prime}(x)\right), \varepsilon^{\prime}(x) \rightarrow 0$ quand $x \rightarrow 0 ; y=e^{-1 / v} ; x=e^{-A / u^{\mu}}$. L'équation $f(x, y)=0$ s'écrit

$$
\sum a_{\alpha, \beta, \gamma, \delta} u^{\alpha} v^{\beta} e^{-\gamma / u^{\mu}} e^{-\delta / v}=0 \quad(\alpha, \beta, \gamma, \delta \text { réels; } \gamma, \delta \geq 0) .
$$

Considérons dans cette somme les termes de multiplicité minimale, quand $(x, y)$ décrit la branche considérée; pour deux tels termes associés à $(\alpha, \beta, \gamma, \delta)$ et $\left(\alpha^{\prime}, \beta^{\prime}, \gamma^{\prime}, \delta^{\prime}\right)$ on a, compte tenu de la forme de l'équation minimale,

$$
\begin{aligned}
& \gamma=\gamma^{\prime} ; \delta=\delta^{\prime} ; \quad \alpha+\beta=\alpha^{\prime}+\beta^{\prime} \quad \text { si } \mu \neq 1, \\
& \gamma+\delta=\gamma^{\prime}+\delta^{\prime} ; \quad \alpha+\beta=\alpha^{\prime}+\beta^{\prime} \quad \text { si } \mu=1 \text {. }
\end{aligned}
$$


Supposons $\mu \neq 1$ (le cas $\mu=1$ se traiterait de manière analogue); notons $\gamma_{0}$ (resp. $\delta_{0}$ ) la valeur commune à tous ces $\gamma($ resp. $\delta)$; on peut écrire l'équation $f(x, y)=0$ sous la forme

$$
P(u, v) e^{-\gamma_{0} / u^{\mu}} e^{-\delta_{0} / v}+Q(u, v)=0
$$

où $P(u, v)$ est une fonction analytique en des puissances éventuellement irrationnelles de $u$ et $v$ et $Q(u, v)$ est de la forme

$$
\sum a_{\alpha, \beta, \gamma, \delta} u^{\alpha} v^{\beta} e^{-\gamma / u^{\mu}} e^{-\delta / v}
$$

le quotient de chaque $e^{-\gamma / u^{\mu}} e^{-\delta / u}$ par $e^{-\gamma_{0} / u^{\mu}} e^{-\delta_{0} / u}$ étant une fonction $C^{\infty}$ plate en 0 (i.e. si $\mu \geq 1,(\gamma, \delta)>\left(\gamma_{0}, \delta_{0}\right)$ pour l'ordre lexicographique). Visiblement, si $v=\varphi(u)$ est l'équation de la branche, $P(u, \varphi(u))$ est plate en 0 . Compte tenu des résultats du $\S 2$, il existe donc une solution $v=\bar{\varphi}(u)$ de l'équation $P(u, v)=0$ avec $\bar{\varphi}(u)=u+\ldots$ fonction analytique de puissances de $u$, telle que $\varphi(u)-\bar{\varphi}(u)$ soit plate en 0 . Notons $\psi(u)$ le "polynôme" de degré $\leq 1$ tel que $\bar{\varphi}(u)=u(1+\psi(u))+u^{2} \varepsilon(u), \varepsilon(u) \rightarrow 0$ quand $u \rightarrow 0$, $\psi(0)=0$; faisons le changement de variables $v=u(1+\psi(u))+u^{2} \omega$; les $v^{\beta}$ et $e^{-\delta / v}$ deviennent analytiques en $\omega$ (cf. 3.7.4) et l'on termine de la manière habituelle : d'après le théorème de préparation analytique, on peut supposer que l'équation en $u, \omega$ est un polynôme distingué en $\omega$ et l'on résout en $\omega$ en utilisant le lemme de Puiseux (§2). On en déduit que $\omega$, donc $v$, admet un développement en un nombre fini de $u^{\alpha} e^{-\gamma / u^{\beta}}$, i.e. $-1 / \log y$ admet un développement en un nombre fini de $(\log 1 / x)^{\alpha} \exp \left(-\gamma(\log 1 / x)^{\beta}\right)$.

P.S. En Mars 94, Lou van den Dries m'a signalé que le théorème 2.1 a été démontré par des méthodes logiques et parallèlement par Chris Miller (Expansions of the real field with power functions, à paraître dans Ann. Pure Appl. Logic). La méthode ici utilise des éclatements très simples que l'on peut expliciter. Elle permet de montrer que, après éclatements, l'adhérence ou chaque composante connexe d'un semi-analytique au sens de 2.1 sont encore semi-analytiques, cf. 2.7 .

\section{Bibliographie}

[1] J. Bochnak, M.-F. Coste-Roy et M. Coste, Géométrie algébrique réelle, Springer, 1987.

[2] R. Moussu et C. A. Roche, Théorie de Khovanskiǔ et problème de Dulac, Invent. Math. 109 (1991), 431-441.

[3] J.-P. Ressayre, Integer parts of real closed exponential fields, in: Arithmetic, Proof Theory and Computational Complexity, Oxford Univ. Press, New York, 1993.

[4] Ta Lê Loi, On the global Eojasiewicz inequalities for the class of analytic logarithmicexponential functions, C. R. Acad. Sci. Paris 318 (1994), 543-548.

[5] J.-C. Tougeron, Sur les ensembles semi-analytiques avec conditions Gevrey au bord, Ann. Ecole Norm. Sup. (4) 27 (1994).

[6] -, Inégalités de Eojasiewicz globales, Ann. Inst. Fourier (Grenoble) 41 (4) (1991), 841865.

[7] A. J. Wilkie, Model completeness results for expansions of the real field II: The exponential functions, manuscript, Oxford, 1991. 